\title{
2D Digital Image Correlation for Sub nm MEMS Measurements
}

\author{
Ryan Adderson ${ }^{1}$ and Ted Hubbard ${ }^{1 *}$ \\ ${ }^{1}$ Mechanical Engineering, Dalhousie University, Halifax NS, Canada \\ *ted.hubbard@dal.ca
}

\begin{abstract}
This paper demonstrates the use of two-dimensional Digital Image Correlation techniques to measure the motion of MEMS devices to sub $\mathrm{nm}$ repeatability. A series of micro photographic images is obtained and by correlating each subsequent image with respect to the initial image, $\mathrm{nm}$ scale displacements can be measured in both X \& Y directions.
\end{abstract}

\section{Keywords; MEMS, DIC, Digital Image Correlation}

\section{INTRODUCTION}

Micro Electro Mechanical Systems or MEMS refers to microscopic devices with features on the order of microns. MEMS are typically manufactured on a substrate such as silicon or glass using modified microelectronics fabrication technologies including photolithography and chemical etching. MEMS devices can include moving parts, sensors and actuators. The motions of MEMS devices can be on the order of $\mu \mathrm{m}$ to $\mathrm{nm}$,

To characterize such small motions, optical measurement using microscopy is often carried out. Measurement resolution is limited by a number of factors including optical performance and camera specifications. Depending on the system, the effective pixel size can be 10's to 100 's of nm. However, the Rayleigh criteria limits the resolution of any system to approximately half the optical wavelength. For visible light this corresponds to a resolution limit of $\sim 250 \mathrm{~nm}$. No improvement of conventional optics or camera (regardless of pixel size) can exceed this resolution limit. Raleigh limits the resolving power, the ability to distinguish two closely spaced objects. It does not limit the ability to measure the motion of a single object. By using large numbers of pixels over an extended area, it is possible to measure motion or displacement to a much finer resolution. By using the aggregate properties of hundreds or thousands pixels, it is possible to measure sub pixel displacements, down to the $\mathrm{nm}$ and even sub $\mathrm{nm}$ level.

A number of researchers have applied sub pixel methods to measure MEMS motion. MEMS motion measurements are often of one dimensional (1D) motion, along a specific direction. For example Ya'akobovitz et al [1] used an edge tracking algorithm to measure 1D displacements to within 1/25 of a pixel, (14 nm resolution for a $400 \mathrm{~nm}$ pixel size). Yamahata et al [2] used Fast Fourier methods to measure 1D displacements of electrostatic actuators to within $1 / 500$ of a pixel $(0.13 \mathrm{~nm}$ resolution for a $\sim 75$ $\mathrm{nm}$ pixel size). Kokorian et al [3] used spline fitting methods to measure the relative 1D motions between a fixed and moving edge to within $1 / 500$ of a pixel $(0.06 \mathrm{~nm}$ resolution for a $\sim 32 \mathrm{~nm}$ pixel size). Adderson et al [4] used image correlation methods to measure 1D displacements of thermal actuators to within $1 / 1600$ of a pixel, $(0.1 \mathrm{~nm}$ resolution for a $\sim 160 \mathrm{~nm}$ pixel size $)$. The high 1D sub pixel resolutions listed above are only possible through the averaging of a large number of pixels. Typical image capture sizes were hundreds of pixels across, thus tens of thousands of pixels contributed to the final result.

The above MEMS examples are 1D. In macroscopic nonMEMS fields such as Civil Engineering Digital Image Correlation (DIC) is widely used to determine two dimensional (2D) deformations and strains. DIC compares a stressed image to an unstressed image and uses image correlation to deduce the applied strain. Typically, a random speckle pattern is pre-applied to the structure to increase image contrast and improve resolution. In a generalized 2D strain application both axial and shear loads may be present and multiple image changes may occur: for example simultaneous translation, stretching and rotation. Thus, the solution is complex and iterative solutions are typically used. Nonetheless sub pixel resolution is possible with macroscale 2D DIC.

DIC has been also used to measure MEMS devices. Berfield et al [5] used DIC to measure nano particle speckle coated polymers to within $1 / 30$ of a pixel ( $7 \mathrm{~nm}$ resolution for a $\sim 210$ nm pixel size). Copeland et al [6] used 2D DIC to measure a nano particle speckle coated thermal actuator to within $1 / 90$ of a pixel (1.4 nm resolution for a $\sim 130 \mathrm{~nm}$ pixel size). In the above MEMS DIC cases, and also in Yagnamurthy et al [7] and Robin et al [8], the original MEMS had separate nano-scale speckle patterns deposited post fabrication. In this paper we present the use of 2D DIC methods as applied to non-speckle patterned MEMS.

\section{2D DigitAL IMAGE CORRELATION}

\section{A. 2D image correlation}

This section will present 2D digital image correlation and will be limited to 2D displacements in $\mathrm{X} \& \mathrm{Y}$ with translation only, no rotation. Consider two images $A \& B$, each $M x N$ pixels in size. For the purposes of this study a test image was constructed consisting of $200 \times 200$ pixels with an intensity of the form $\sin \left(x / \lambda_{x}\right) * \sin \left(y / \lambda_{y}\right)$ where $\lambda_{\mathrm{i}}$ refers to the spatial 
wavelength. Note that although the test image is periodic, this is not required, and similar results can be obtained with a nonperiodic test image. Negative values were set to zero and a small amount of random noise (5\%) was added to the image. The test image is shown in Fig 1 left. As this is a mathematical function it can be shifted an arbitrary number of pixels in $x$ and $y$. Fig. 1 right shows the original image shifted by $\left(\delta_{\mathrm{x}}, \delta_{\mathrm{y}}\right)=(8.21,4.73)$ pixels.
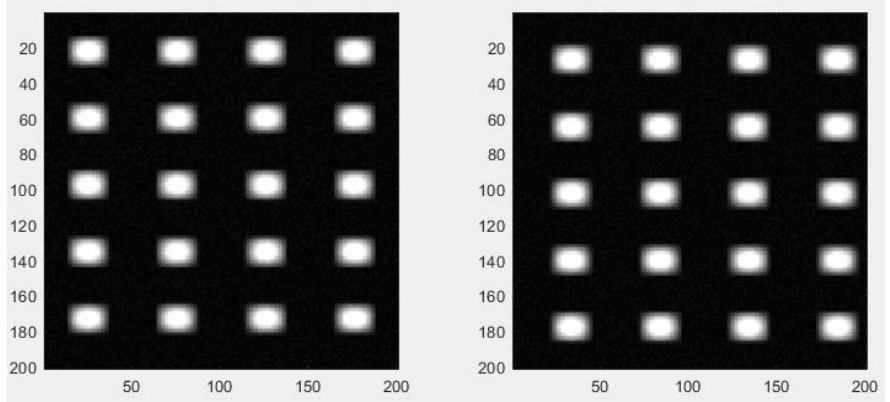

Figure 1. Test 2D image, 200x200 pixels. LEFT: original image, RIGHT: image shifted in $(\mathrm{x}, \mathrm{y})$ by $(8.21,4.73)$ pixels.

To measure image shift we can use cross correlation, which is based on image correlation. The image correlation of $A$ with respect to $B$ is calculated by multiplying each element of $A$ by its corresponding element in $B$ and summing all the products. The cross correlation uses image correlation but with imposed image shifts. Cross correlation $(A \star B)$ is obtained by shifting $A$ by amounts $\delta_{\mathrm{x}}$ and $\delta_{\mathrm{y}}$ and correlating the shifted $A$ with $B$ :

$(A \star B)\left[\delta_{x}, \delta_{y}\right]=\sum_{j=1}^{N} \sum_{i=1}^{M} A\left(i+\delta_{x}, j+\delta_{y},\right) \times B(i, j)$

A $2 M x 2 N$ correlation matrix is formed from the calculated correlation values for all possible $\delta_{x}, \delta_{y}$ shifts of $A$. The peak of the correlation matrix is the image shift. Note that as the image is shifted to the right, the rightmost pixels exceed the matrix size and are dropped, new zero value pixels are added on the left, similarly for pixels shifts on the left, top and bottom. Thus, for large image shifts correlation matrix values decrease.

The software MATLAB includes a function $x$ corr 2 which performs the 2D cross correlation on two matrices. Fig. 2 shows a portion of the MATLAB generated correlation matrix for Fig. 1 images. The highest peak indicates the image shift $\left(\delta_{\mathrm{x}}, \delta_{\mathrm{y}}\right)$. Note the slight shift in $x \& y$ of the peak position. Note also that there are secondary surrounding peaks: these correspond to shifts by $\lambda_{x}$ or $\lambda_{y}$ and appear in any periodic image. The secondary peaks are smaller due to dropped edge pixels.

Fig 3 top shows the central peak region only. The calculated image shift is $(8,5)$ pixels. Cross correlation can only achieve pixel level accuracy. To obtain sub-pixel values, the central region can be subdivided into smaller increments (e.g. 512 or 1024) and the intermediary values calculated using a spline interpolation. The peaks of the splined region can then be found with sub pixel accuracy ( 0.01 pixels or better are possible). Fig. 3 bottom shows the spline peak with calculated shift of (8.205, $4.734)$ pixels. These values differ slightly from the true values by $(0.005,0.004)$ pixels due to the $5 \%$ noise in the images.

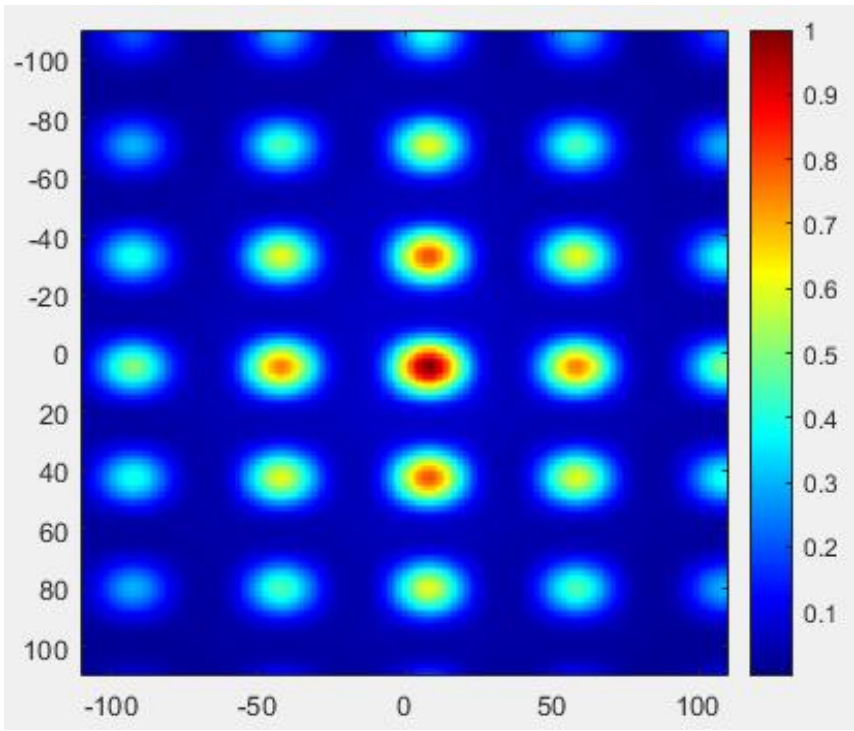

Figure 2. Central portion of MATLAB $x \operatorname{corr} 2$ 2D correlation of Figure 1 images.

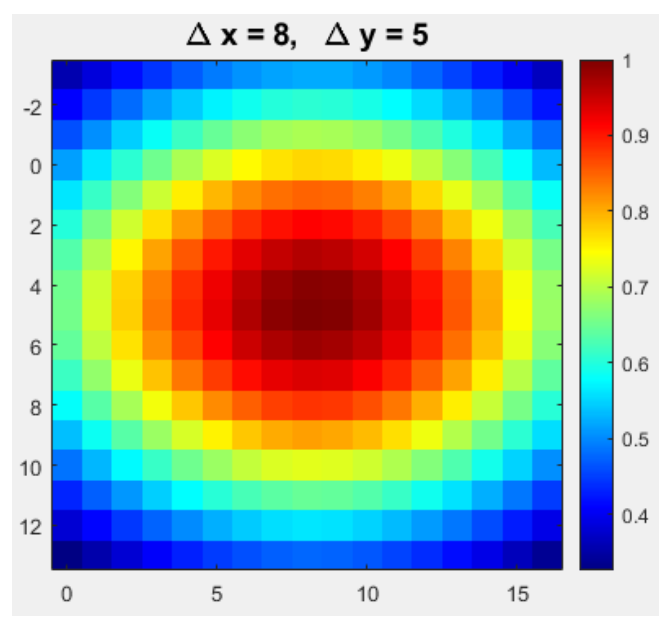

$\Delta x=8.205, \quad \Delta y=4.734$

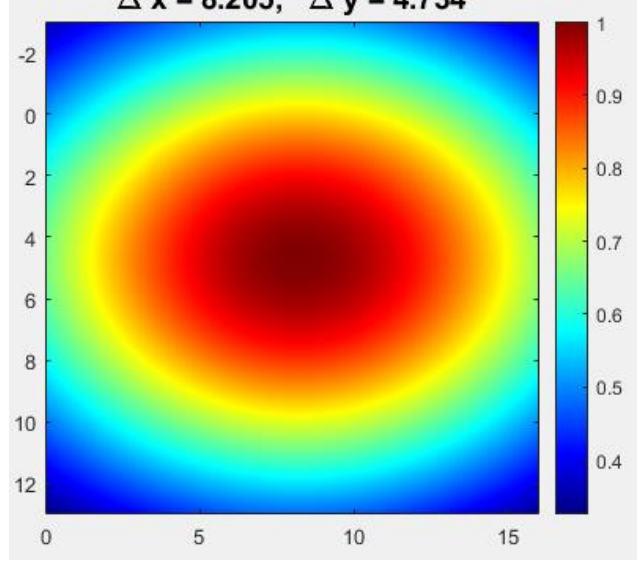

Figure 3. Figure 2 central correlation peak. TOP: 1 pixel sampling $(\mathrm{x}, \mathrm{y})=$ $(8,5)$, BOTTOM: $1 / 512$ sub pixel spline fit $(x, y)=(8.205,4.734)$. 


\section{B. Resolution factors}

Real photographic images will be more complex than the idealized images presented above. In the following section effects such as noise levels and image properties will be discussed by applying degradations to the Fig. 1 test images. Firstly, the correlations for Fig. 1 with an input shift of (8.21, 4.73) pixels were run 20 times with a random noise level of $5 \%$. The average values of the $(\mathrm{x}, \mathrm{y})$ errors were: $(-0.005 \pm 0.003,0.002 \pm 0.002)$ pixels. Next the same correlations were run 20 times for various degradations to both Fig. 1 left and right images. Fig. 4 shows the test image from Fig. 1 left along with various image degradations; Table 1 lists the resultant errors.
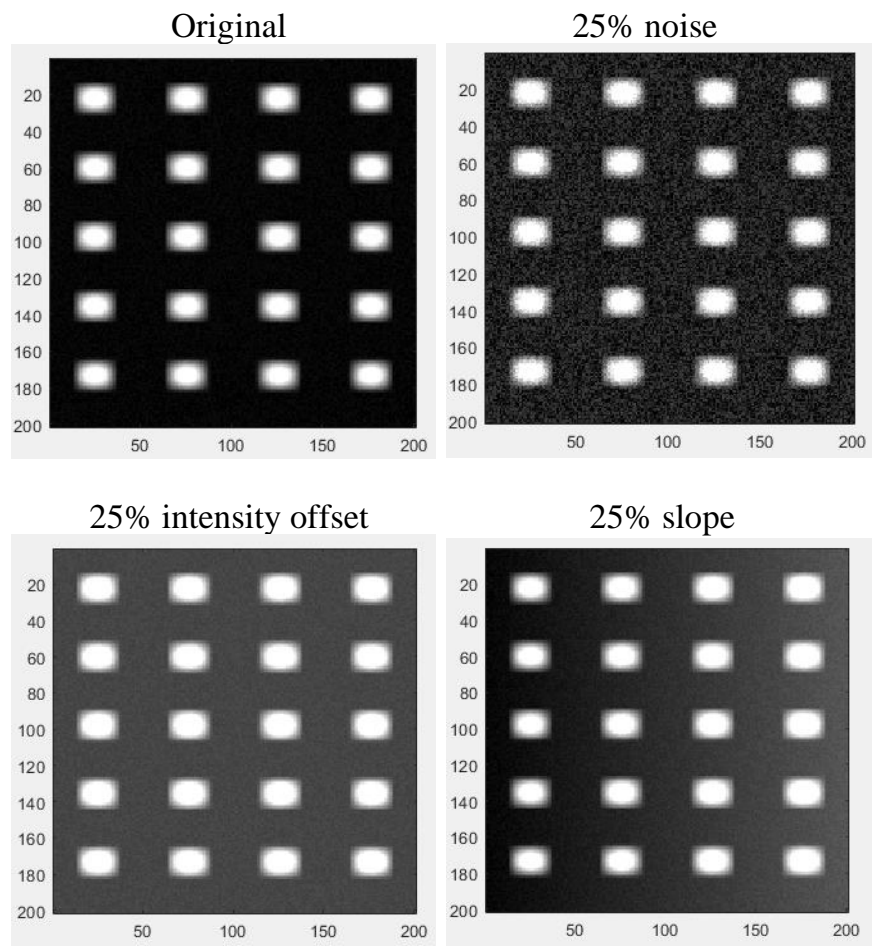

Figure 4. Image degradations of Fig 1 left.

TABLE I. EFFECT OF IMAGE DEGRADATION

\begin{tabular}{|l|c|c|}
\hline & Error x & Error y \\
\hline Original (5\% noise) & $-0.005+/-0.003$ & $0.002+/-0.002$ \\
\hline Noise 25\% & $-0.051+/-0.020$ & $-0.026+/-0.022$ \\
\hline Offset 25\% & $-0.277+/-0.003$ & $-0.153+/-0.003$ \\
\hline Slope 25\% & $-0.235+/-0.004$ & $-0.089+/-0.002$ \\
\hline
\end{tabular}

Noise: As shown in Fig. 4 upper right, the noise level may be larger than in the original test images. Increasing image noise will degrade the resolution. The effect of noise is especially detrimental on small or low contrast images. Choosing large regions will reduce the effects of noise: for an image capture area of $L x W$ pixels in size, the resolution is proportional to $1 / \sqrt{L W}$ [4]. Similarly choosing regions with high contrast and steep image gradients will reduce the effects of noise and sub 0.01 pixel resolution is still achievable with noisy images.

Offset: Real camera images do not show true black, even the darkest parts of an image will have some intensity offset value. As shown in Fig. 4 lower left, the minimum pixel value is offset from zero by some positive amount. Sub 0.01 pixel resolution is not achievable when image intensity offset is present. The cause is the dropping of edge pixels: if any offset is present, then the dropped pixels be replaced with zeros on the opposite side and this will skew the correlation matrix, leading to significant errors. When offset is present even 0.1 pixels accuracy may not be possible. However, this is easily addressed by subtracting any offset value from the images before correlation. This is essential for sub pixel accuracy.

Slope: Uneven illumination can cause a slope in the image intensity. For example, as shown in Fig. 4 lower right, the left side might be brighter than the right. As with offset this will cause significant errors and must be removed for sub pixel accuracy.

With careful control of image noise, image contrast, and by de-offsetting and de-sloping of images, sub 0.01 pixel resolution can be achieved.

\section{EXPERIMENTAL SETUP}

The MEMS devices used in this study were fabricated using the commercially available surface micromachining process PolyMUMPs (Polysilicon Multi User MEMS process) [9]. PolyMUMPS is a three layer Polysilicon fabrication technology with feature sizes of $\sim 2-3 \mu \mathrm{m}$, and layer thicknesses of $\sim 0.5-2 \mu \mathrm{m}$. In this study electrothermal actuators were used as the motive device. One common type of thermal actuator is the bent beam or 'chevron' type actuator $[10,11]$. The beams are anchored at both ends and bent in the middle. Current passes through the beams via the anchors, causing the beams to heat up and expand. The bend in the chevron amplifies the expansion and causes a $\sim 10 \mathrm{x}$ transverse motion. Typical input values are a few volts and several milliamps current. The output motion ranges from nm to a few $\mu \mathrm{m}$ and is proportional to input electrical power [10].

Micro photographs of the MEMS devices were captured using a probestation (Wentworth M901) and camera setup mounted on a vibration isolation table. The microscope objective used was 50x with zoom 2. The camera used was Point Grey Research Colour Firewire GRAS-14S (1280x960 pixels, $0.163 \mu \mathrm{m} /$ pixel). Fig. 5 shows a typical MEMS image, the device was part of cell squeezer developed by Barazani et al [11] but here is used solely as a motion device. The red section moves to the right, the yellow section is fixed. The device was fabricated with long vertical bars, specifically added to increase the optical contrast of the structures. A function generator (Keysight 33210A) was used to drive thermal actuators. Both the function generator and camera were controlled through a Labview program which ran through an automated sequence of setting the voltage, talking $\mathrm{N}$ images and then moving to the next voltage. 
The resultant saved images were then analyzed using a MATLAB program that performed the 2D DIC. Within the MATLAB program, the user selects a Region of Interest (ROI) on the moving device. This ROI is shown as a red box in Fig. 5. Each Image 1 to $\mathrm{N}$ is: 1) cropped to the ROI, 2) converted to grayscale double, 3) de-offset and de-sloped as discussed in Section II. Careful selection of as large a ROI as possible with uniform edge brightness is critical to achieving sub 0.01 pixel resolution. Each processed Image 1 to $\mathrm{N}$ is then cross correlated with processed Image 1 using xcorr 2 and a spline peak interpolation to produce a sub pixel determination of the displacement.

However even on a static non-moving MEMS device, there will be some random sub pixel frame motion caused by vibrations. Depending on the mechanical isolation, this can be several $\mathrm{nm}$ to 10 's of $\mathrm{nm}$. To cancel this effect, in the MATLAB code also asks the user to select a second ROI containing a 'fixed' device. This second ROI is shown as a dashed yellow box in Fig. 5. The MATLAB program is then run again on the fixed ROI. Since the fixed device should be stationary, subtracting the 'fixed' measured motion from the moving device results in the net motion of the moving device.

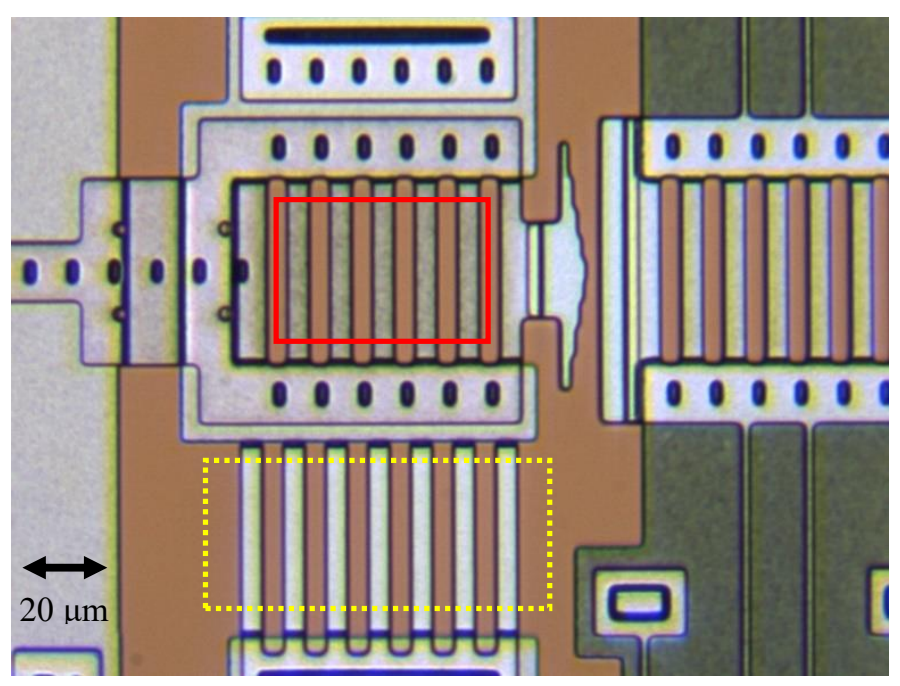

Figure 5. MEMS device. Regions of Interest (ROI) for moving device (solid RED box) and 'fixed' device (dashed yellow box).

\section{RESULTS \& DISCUSION}

As the output of the thermal actuators should increase with increasing voltage, the actuator was first driven in $0.01 \mathrm{~V}$ steps from $0.20 \mathrm{~V}$ to $0.30 \mathrm{~V}$. The motion was aligned along the $\mathrm{X}$ axis of the camera. At each voltage, 10 images were taken and the average and standard deviation calculated. Fig 6 shows the measured displacement X measurements in blue and $\mathrm{Y}$ measurements in red. At this low voltage the expected parabolic actuator response (power $\rightarrow \mathrm{V}^{2}$ ) is close to linear and the $\mathrm{X}$ step increments are approximately the same: $\sim 3-4$ $\mathrm{nm}$. The maximum displacement was $40 \mathrm{~nm}(0.25$ pixel $)$ in $X$ and $-0.5 \mathrm{~nm}(0.003$ pixel) in $\mathrm{Y}$. The average $(\mathrm{X}, \mathrm{Y})$ standard deviations within a step were $\pm(0.17,0.13) \mathrm{nm}(\sim 0.001$ pixel $)$.

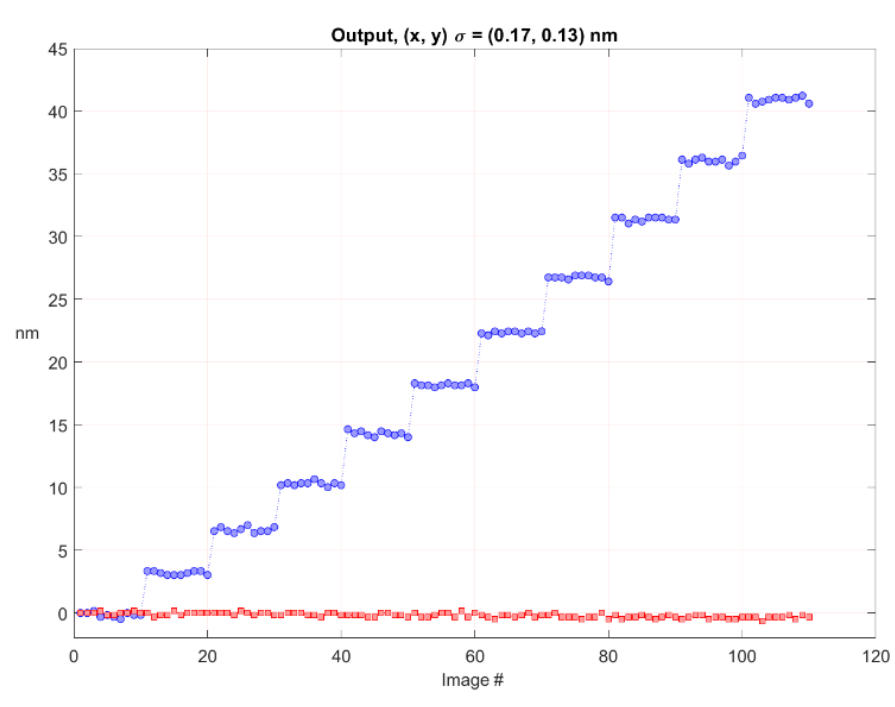

Figure 6. $\mathrm{X}$ direction displacement of chevron actuator vs. voltage: $0.2 \mathrm{~V}$ to $0.3 \mathrm{~V}$ in $0.01 \mathrm{~V}$ steps. Blue is X measurement, red is Y measurement. $\sigma= \pm$ $(0.17,0.13) \mathrm{nm}$.

The above test was repeated with the camera rotated: the motion was now aligned along the $\mathrm{Y}$ axis of the camera as shown in Fig. 7. Fig. 8 shows the measured displacement, $\mathrm{X}$ measurements in blue and $\mathrm{Y}$ measurements in red. The maximum displacements were $\sim 40 \mathrm{~nm}(0.25$ pixel $)$ in $\mathrm{Y}$ and 2 $\mathrm{nm}(0.01$ pixel $)$ in $\mathrm{X}$. The average $(\mathrm{X}, \mathrm{Y})$ standard deviations within a step were $\pm(0.19,0.25) \mathrm{nm}(\sim 0.001$ pixel $)$. Note the slight upward $\mathrm{X}$ drift indicating that the motion was not aligned exactly with the Y axis (as can be observed in Fig. 7).

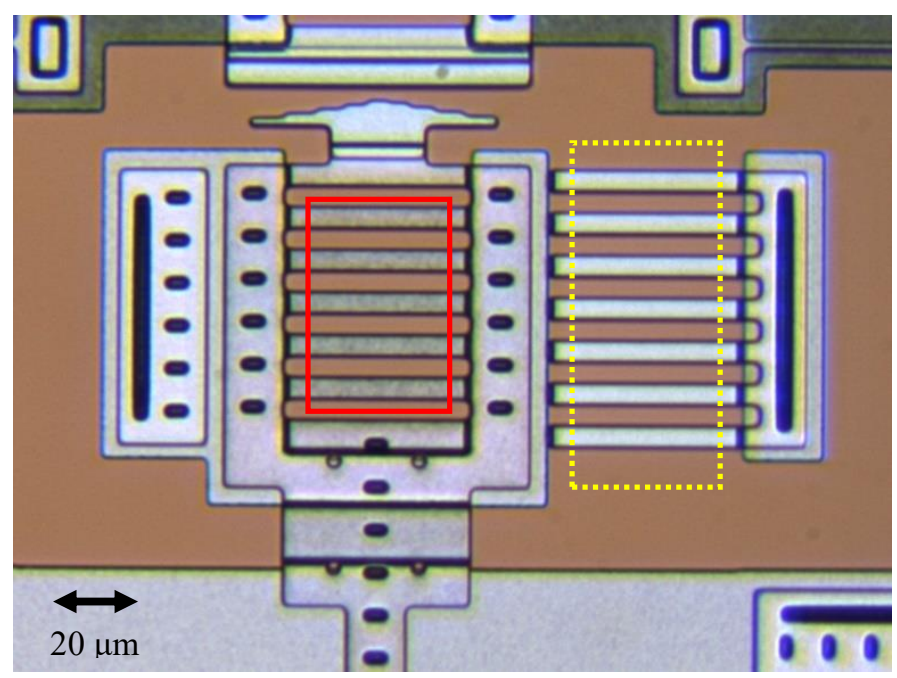

Figure 7. MEMS device oriented for motion along Y axis. ROI's for moving device (solid RED box) and 'fixed' device (dashed yellow box). 


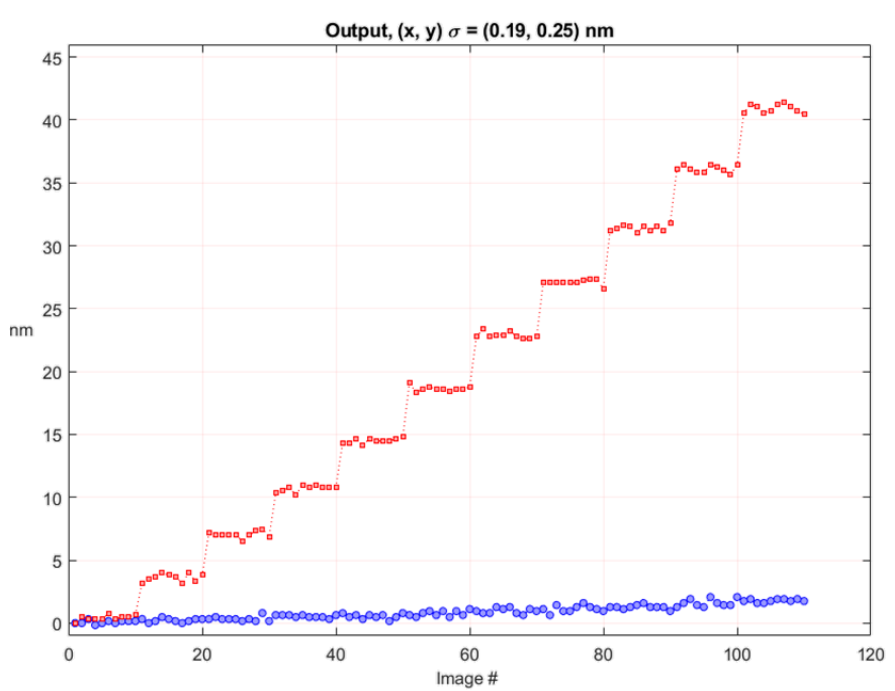

Figure 8. Y direction displacement of chevron actuator vs. voltage. Blue is $\mathrm{X}$ measurement, red is Y measurement. $\sigma= \pm(0.19,0.25) \mathrm{nm}$.

The test was again repeated with camera rotated: the motion was now aligned along an approximately 45 degree axis to the camera as shown in Fig. 9. Note that the selected ROI were smaller and thus larger noise values are expected. Fig. 10 shows the measured displacement, $\mathrm{X}$ measurements in blue and $\mathrm{Y}$ measurements in red. The $\mathrm{X} \& \mathrm{Y}$ amplitudes are both reduced as the motion is at 45 degrees. The maximum displacements in $\mathrm{X}$ and $\mathrm{Y}$ were $\sim 28 \mathrm{~nm}(0.25$ pixel $)$. The $\sin$ and $\cos$ of $45^{\circ}$ are 0.7 and $40 \mathrm{~nm} x 0.7=28 \mathrm{~nm}$. The average $(\mathrm{X}, \mathrm{Y})$ standard deviations within a step were $\pm(0.49,0.66)$ nm, ( 0.004) pixels.

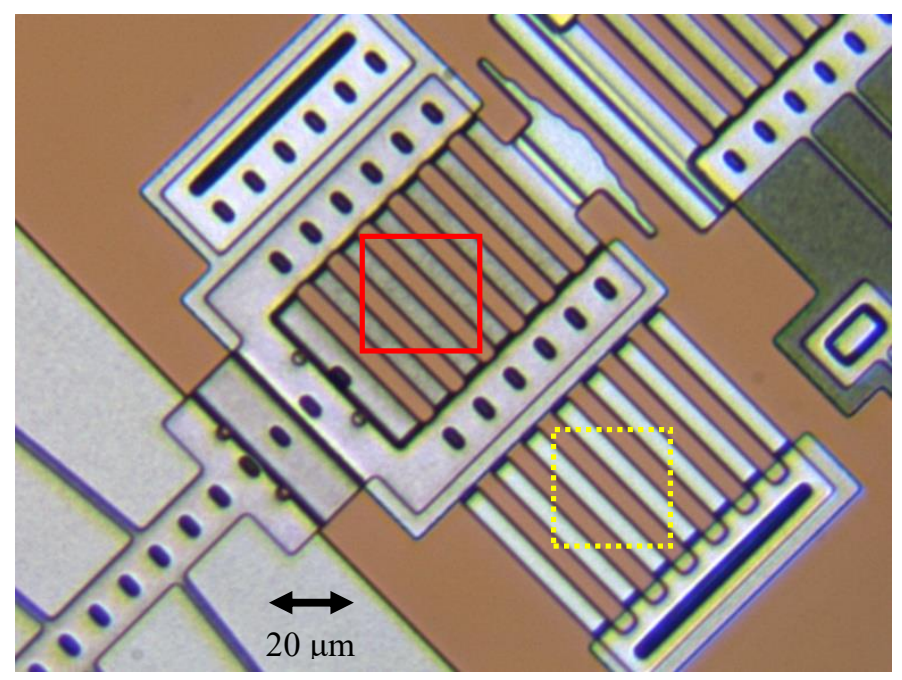

Figure 9. MEMS device oriented for motion along $45^{\circ}$ axis. ROI's for moving device (solid RED box) and 'fixed' device (dashed yellow box).

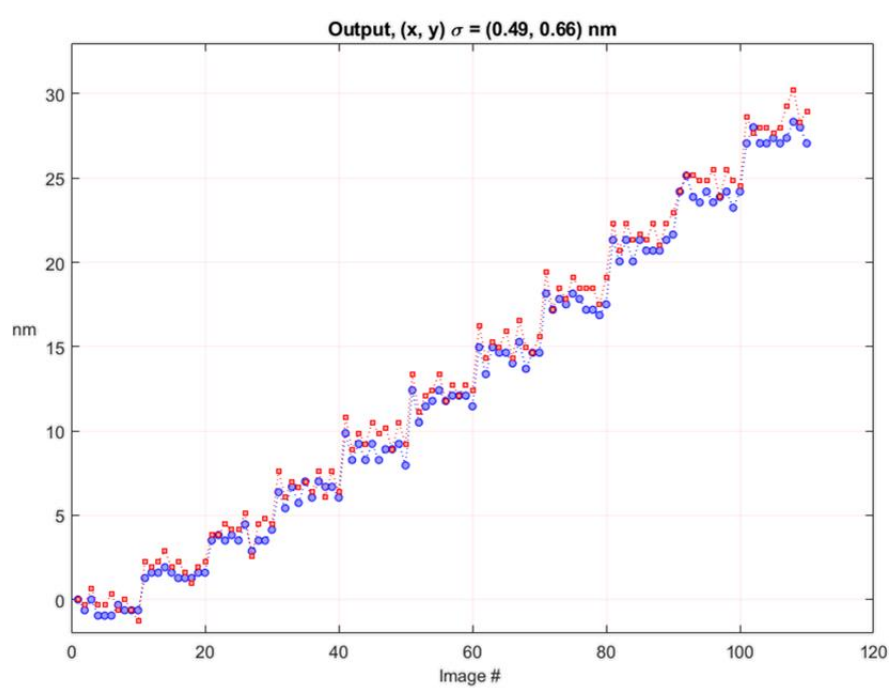

Figure $10.45^{\circ}$ displacement of chevron actuator vs. voltage. Blue is $\mathrm{X}$ measurement, red is $\mathrm{Y}$ measurement. $\sigma= \pm(0.49,0.66) \mathrm{nm}$.

Next repeated small alternating voltage steps were input and the 10 images taken at each voltage. Applying alternating voltages of $0.200 \mathrm{~V}$ and $0.215 \mathrm{~V}$ produced a step motion of $\sim 5$ $\mathrm{nm}$. By rotating the camera on the microscope images were captured aligned with different directions. Fig. 11 shows the results for $\mathrm{X}$ motion, The average $(\mathrm{x}, \mathrm{y})$ standard deviations at each voltage were $(0.20,0.17) \mathrm{nm}$. Fig. 12 shows the results for Y motion. The average (x, y) standard deviations at each voltage were $(0.18,0.21) \mathrm{nm}$. Fig. 13 shows the results when aligned at 45 degrees: average (x, y) standard deviation at each voltage of $(0.37,0.57) \mathrm{nm}$. The X \& Y amplitudes are both reduced as the motion is at 45 degrees. Note the increased noise $\mathrm{nm}$ in both $\mathrm{X}$ and $\mathrm{Y}:(0.49,0.49) \mathrm{nm}$, this is due to smaller ROI's being used to fit inside the now diamond oriented device.

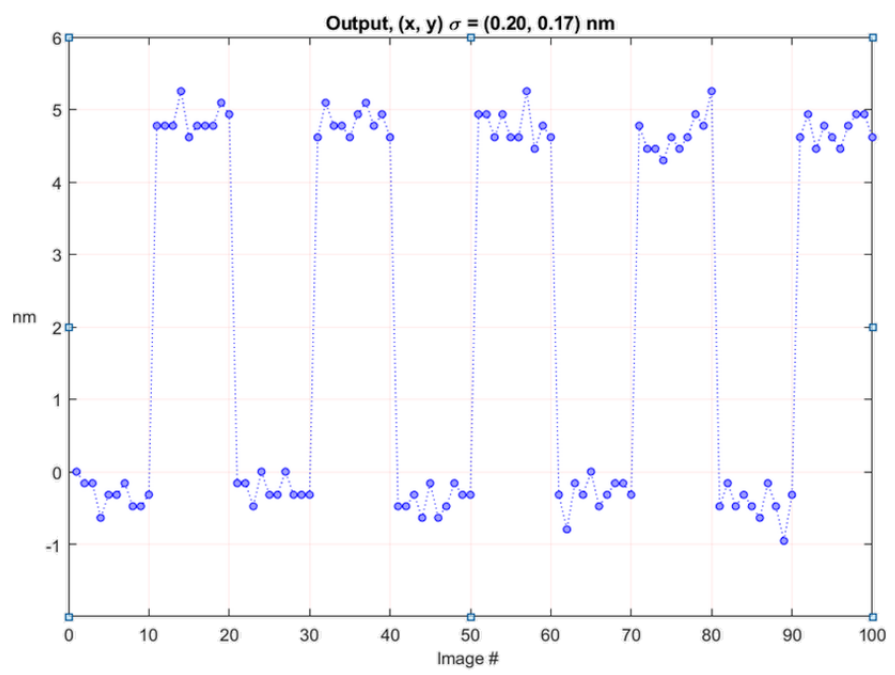

Figure 11. X step detection. $5 \mathrm{~nm}$ steps, $\sigma= \pm(0.20,0.17) \mathrm{nm}$. 


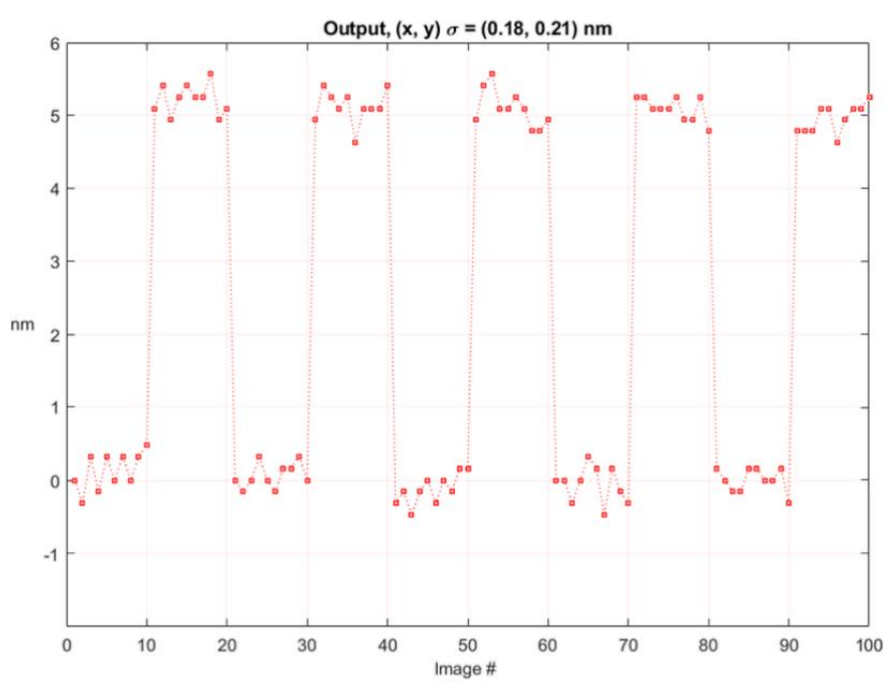

Figure 12. Y step detection. $5 \mathrm{~nm}$ steps, $\sigma= \pm(0.18,0.21) \mathrm{nm}$.

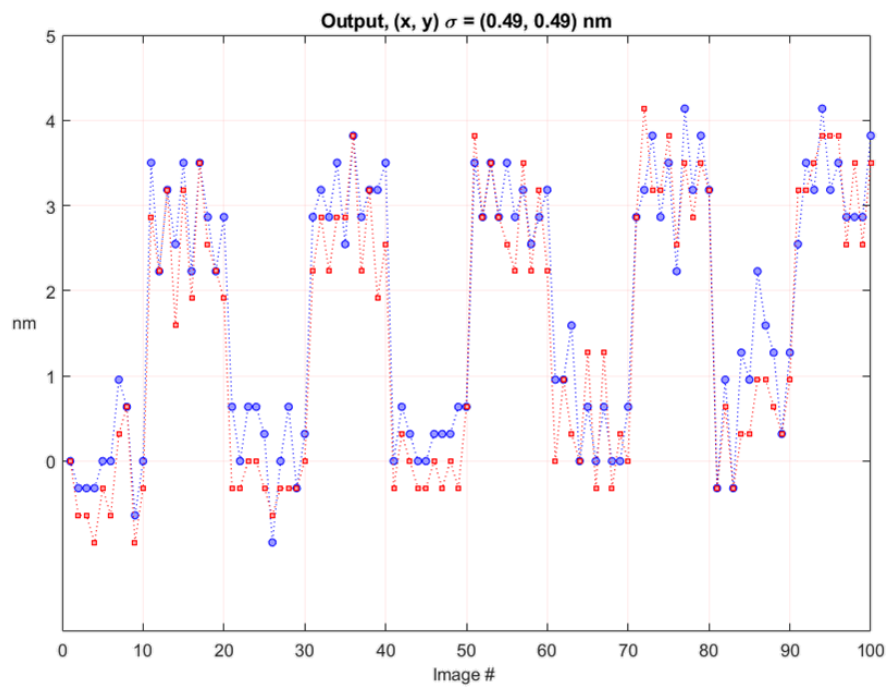

Figure 13. 45 degree step detection, $\sigma= \pm(0.49,0.49) \mathrm{nm}$.

\section{CONCLUSIONS}

We have described a 2D image correlation method for comparing the motion of successive MEMS photographic images and determining the MEMS motion to a high resolution. A MATLAB program cross correlates user selected Regions Of Interest within the MEMS images. MEMS motion in $\mathrm{X}, \mathrm{Y}$ and 45 degrees axes was measured, and sub $\mathrm{nm}$ resolution corresponding to sub 0.01 pixels was achieved

\section{ACKNOWLEDGMENT}

This work was funded by the NSERC - Natural Sciences and Engineering Research Council of Canada, and fabrication services were obtained through CMC - Canadian Microsystems Corporation.

\section{REFERENCES}

[1] A. Ya'akobovitz, D. Copic, J. Beroz and A. Hart, "Nanoscale displacement measurement of microdevices via interpolation-based edge tracking of optical images", Journal of Micromechanics and Microengineering, vol. 23, no. 4, 2013. DOI: 10.1088/0960$1317 / 23 / 4 / 045004$

[2] C. Yamahata, E. Sarajlic, G. Krijnen and M. Gijs, "Subnanometer Translation of Microelectomechanical Systems Measured by Discrete Fourier Analysis of CCD Images", Journal of Micromechanics and Microengineering, vol. 19, no. 5, pp. 1273-1275, 2010. DOI: 10.1109/JMEMS.2010.2067445

[3] J. Kokorian, F. Buja and W. van Spengen, "In-Plane Displacement Detection With Picometer Accuracy on a Conventional Microscope", Journal of Microelectromechanical Systems, vol. 24, no. 3, pp. 618-625, 2015. DOI: 10.1109/jmems.2014.2335153

[4] R. Adderson and T. Hubbard, "Millipixel image correlation for sub nm measurement of MEMS motion", Journal of Micromechanics and Microengineering, vol. 29, no. 11, DOI: 10.1088/1361-6439/ab3ede

[5] T. Berfield et al. "Micro- and Nanoscale Deformation Measurement of Surface and Internal Planes via Digital Image Correlation", Experimental Mechanics, vol. 47, pp. 51-62, 2007. DOI: 10.1007/s11340-006-0531-2

[6] C. Copland et al. "Characterization Of Electrothermal Actuation With Nanometer And Microradian Precision”, Transducers 2015, Anchorage, AK, June 21-25, 2015, DOI: 10.1109/TRANSDUCERS.2015.7181042

[7] S. Yagnamurthy et al, "Mechanical and Ferroelectric Behavior of PZTBased Thin Films", Journal of Microelectromechanical Systems, vol. 20, no. 6, pp. 1250-1258, 2011. DOI: 10.1109/jmems.2011.2167666

[8] C. Robin, A. Vishnoi and K. Jonnalagadda, "Mechanical Behavior and Anisotropy of Spin-Coated SU-8 Thin Films for MEMS", Journal of Microelectromechanical Systems, vol. 23, no. 1, pp. 168-180, 2014. DOI: 10.1109/jmems.2013.2264341

[9] J. Carter et al, PolyMUMPs Design Handbook, 11th ed. 2005

[10] R. Hickey, M. Kujath and T. Hubbard, "Heat transfer analysis and optimization of two-beam microelectromechanical thermal actuators", Journal of Vacuum Science \& Technology A: Vacuum, Surfaces, and Films, vol. 20, no. 3, pp. 971-974, 2002. DOI: 10.1116/1.1468654.

[11] B. Bazarani, S. Warnat, A. Fine and T. Hubbard, "MEMS squeezer for the measurement of single cell rupture force, stiffness change, and hysteresis", Journal of Micromechanics and Microengineering, vol. 27, no. 2, pp. 50-58, DOI: 10.1088/1361-6439/27/2/025002 